\title{
A clinical retrospective study comparing thoracic epidural catheterization between awake and anesthetized patients
}

Received May 31, 2018

Revised 1st, July 16, 2018

2nd, August 23, 2018

Accepted September 1, 2018

\section{Corresponding author}

Tae-Yun Sung, M.D., Ph.D.

Department of Anesthesiology and

Pain Medicine, Konyang University

Hospital, Konyang University College

of Medicine, 158 Gwanjeodong-ro,

Seo-gu, Daejeon 35365, Korea

Tel: 82-42-600-9316

Fax: 82-42-600-9398

E-mail: unt1231@naver.com

\section{ORCID}

https://orcid.org/0000-0002-0714-1477

\section{Seok-Jin Lee, Sung-Ae Cho, Chi Bum In, Tae-Yun Sung, and Po-Soon Kang}

Department of Anesthesiology and Pain Medicine, Konyang University Hospital, Konyang University College of Medicine, Daejeon, Korea

\begin{abstract}
Background: The clinical outcomes and safety of thoracic epidural catheterization in anesthetized adult patients has not yet been established. The purpose of this study was to compare clinical differences between epidural catheterization performed before and after anesthesia for postoperative pain control.
\end{abstract}

Methods: The medical records of 549 patients who received thoracic epidural catheterization before (awake group, $n=303$ ) or after (anesthetized group, $n=246$ ) induction of anesthesia for major abdominal surgery were reviewed retrospectively.

Results: The catheter insertion time (1.6 \pm 1.5 vs. $1.1 \pm 1.2 \mathrm{~min}$; 95\% confidence interval [95\% Cl], 0.3-0.8; effect size, 0.368; $\mathrm{P}<0.001$ ) and number of attempts required for successful epidural catheterization $(1[1,3]$ vs. 1 [1, 2], $P=0.003$ ) were increased in the awake group. The incidence rates of dural puncture, vascular injury and postoperative paresthesia were similar between the two groups. The median surgical site numerical rating scale pain score $(0=$ no pain, $10=$ worst pain imaginable) was lower in the awake group than in the anesthetized group (3 vs. 4 on postoperative day 1, $\mathrm{P}<0.001$; and 2 vs. 3 on postoperative day $3, P=0.002$ ). Serious complications, including meningitis, epidural abscess, epidural hematoma, spinal cord injury, and paraplegia, were not observed in either group.

Conclusions: Successful epidural catheterization before induction of anesthesia required more attempts versus after anesthesia. Overall complication rates of thoracic epidural catheterization were similar regardless of the timing of the procedure.

Keywords: Catheterization; Epidural analgesia; Postoperative complications.

\section{INTRODUCTION}

Thoracic epidural anesthesia and analgesia is not only the most effective type of analgesia, but also decreases the time required for tracheal extubation, the duration of intensive care unit (ICU) stay, levels of serum stress hormones, and the consumption of opioids within 24 hours after operation [1-3]. In addition, thoracic epidural analgesia positively impacts on intestinal recovery, dietary intake, quality of life and mortality rate after major abdominal surgery $[4,5]$.

In pediatric cases, the efficacy and safety of regional analgesia, including for caudal, lumber and thoracic epidural catheterization, has been well established, with such anesthesia being commonly applied in the context of deep sedation or general anesthesia $[6,7]$. However, thoracic epidural catheterization for anesthetized adult patients is still contro-

This is an Open Access article distributed under the terms of the Creative Commons Attribution Non-Commercial License (http://creativecommons.org/licenses/by-nc/4.0) which permits unrestricted non-commercial use, distribution, and reproduction in any medium, provided the original work is properly cited.

Copyright (C) the Korean Society of Anesthesiologists, 2019 
versial, due mainly to safety issues [8]. In a previous study analyzing 4,298 anesthetized adult patients, lumbar epidural catheterization carried a small risk of neurological complications [9]. Few studies have compared the clinical efficacy and safety of thoracic epidural catheterization between awake and anesthetized adult patients. This study evaluates whether there are clinical differences in the optimal timing of epidural catheterization (i.e., before vs. after induction of anesthesia) by retrospectively analyzing the medical records of patients who underwent thoracic epidural catheterization for pain control after major abdominal surgery.

\section{MATERIALS AND METHODS}

This retrospective study was approved by the Institutional Review Board (IRB) of our hospital (IRB no. 2017-09-001001). The requirement for informed consent was waived because there was no intervention component, only a chart review. We retrospectively reviewed the routine electronic medical records and handwritten epidural procedure records of patients who underwent thoracic epidural catheter insertion during major abdominal surgery from January 2012 to December 2015. Records were classified according to the presence or absence of anesthesia during catheter insertion: 1) awake group: epidural catheterization performed before general anesthesia; and 2) anesthetized group: epidural catheterization performed after induction of general anesthesia. The exclusion criteria were abnormal blood coagulation, indwelling endotracheal tube on leaving the operating room, transfer to an ICU after surgery, and incomplete medical record documentation.

All patients fasted preoperatively for at least 8 hours. After routine monitoring of noninvasive blood pressure and oxygen saturation, as well as an electrocardiogram, general anesthesia was induced with propofol, rocuronium and remifentanil. Endotracheal intubation was performed in all patients for mechanical ventilation during surgery. Maintenance of anesthesia was achieved using desflurane and remifentanil in all patients.

In the awake group, epidural catheter insertion was performed immediately prior to induction of anesthesia, while epidural catheter insertion was conducted immediately after anesthesia induction in the anesthetized group. All epidural catheterization procedures were performed in the lateral de- cubitus position for the purpose of postoperative analgesia, as induced by an experienced anesthesiologist. For catheterization before general anesthesia, $2-3 \mathrm{ml}$ of $2 \%$ lidocaine was injected at the skin puncture site. An 18-gauge Tuohy needle and 20-gauge epidural catheter were inserted in the T9-12 thoracic region, according to the surgical procedure. In both groups, a paramedian approach and loss of resistance (LOR) technique (using an air-filled glass syringe) were used to approach the epidural space. Catheter placement was tested by injection of 2\% lidocaine with 1:200,000 epinephrine (total, 3 $\mathrm{ml}$ ) after catheter insertion into the epidural space to a depth of $4 \mathrm{~cm}$. Approximately 30 minutes after epidural catheterization, epidural patient-controlled analgesia (PCA; mixture of local anesthetic [ $200 \mathrm{ml}$ of $0.2 \%$ ropivacaine] and opioid [500 $\mu \mathrm{g}$ of fentanyl]) was started at a continuous infusion rate of 3 $\mathrm{ml} / \mathrm{h}$ (bolus of $1 \mathrm{ml}$ and lockout interval of 30 minutes). After surgery, sugammadex, or a combination of pyridostigmine and glycopyrrolate, was injected for reversal of the neuromuscular blockade.

The data obtained during thoracic epidural catheterization were analyzed in terms of: 1) time taken for epidural catheter insertion (from skin puncture with Tuohy needle to removal of the needle after catheterization); 2) number of attempts required for successful epidural catheter insertion; 3 ) ease of catheter insertion (subjective opinion of the practitioner: easy or difficult); and 4) incidence of dural puncture, radicular pain and vascular injury (blood return from Tuohy needle or catheter, either spontaneously or during aspiration) during epidural needle or catheter insertion.

The postoperative data were analyzed in terms of: 1) duration of epidural PCA infusion; 2) pain score on numerical rating scale (NRS; $0=$ no pain, $10=$ worst pain imaginable) for the wound in the operating site on postoperative days 1 and $3 ; 3$ ) incidence of thoracic back pain caused by thoracic epidural placement and maximum NRS thoracic back pain score during hospitalization: 4) minor complications, such as headache, nausea, vomiting, urinary retention, dizziness, pruritus, constipation, or signs of paresthesia including prickling, tingling, or numbness; and 5) serious complications, such as meningitis, epidural abscess, epidural hematoma, or paraplegia. 


\section{Statistical analysis}

All data were analyzed with PASW Statistics software (ver. 18.0 for Windows, IBM Co., USA). The normality of the data distribution was tested using the Shapiro-Wilk test. The data were analyzed using the $t$-test or Mann-Whitney $U$ test for continuous variables, and the chi-squared test or Fisher's exact test for categorical variables, as appropriate. Data are expressed as means \pm standard deviation (SD), medians (1Q, $3 \mathrm{Q})$, percentages, or numbers. $\mathrm{P}<0.05$ was considered statistically significant.

\section{RESULTS}

A total of 602 patients were enrolled, with 53 excluded based on the study exclusion criteria. The final total of patients was 549: 303 were awake during epidural catheterization and 246 were anesthetized.

Demographic data were similar between the awake and anesthetized groups (Table 1). The data on epidural catheterization time and number of attempts required for successful epidural catheterization are shown in Table 2. The procedure time (1.6 \pm 1.5 vs. $1.1 \pm 1.2 \mathrm{~min}$; $95 \%$ confidence interval [95\% CI], 0.3-0.8; effect size 0.368; $\mathrm{P}<0.001$ ) and number of attempts $(1[1,3]$ vs. $1[1,2]$, median $[1 \mathrm{Q}, 3 \mathrm{Q}], \mathrm{P}=0.003)$ were significantly increased in the awake group. The presence of radicular pain during catheterization was noted only in the awake group, where $1.7 \%$ (5/303) of the patients in that group reported pain. The ease of catheter placement, and incidence of dural puncture and vascular injury were similar between the two groups.

Duration of epidural PCA infusion was similar between the groups (Table 3). The NRS pain score for the operation site in the awake group was lower than that in the anesthetized

Table 1. Demographic Data

\begin{tabular}{|c|c|c|c|}
\hline Variable & $\begin{array}{l}\text { Awake group } \\
\qquad(n=303)\end{array}$ & $\begin{array}{l}\text { Anesthetized group } \\
\qquad(n=246)\end{array}$ & $P$ value \\
\hline Sex (male) & $198(65.3)$ & $167(67.9)$ & 0.531 \\
\hline Age $(y r)$ & $63.6 \pm 11.8$ & $63.1 \pm 11.6$ & 0.590 \\
\hline Weight (kg) & $61.5 \pm 10.0$ & $60.9 \pm 11.3$ & 0.346 \\
\hline Height $(\mathrm{cm})$ & $160.8 \pm 8.8$ & $162.1 \pm 8.5$ & 0.087 \\
\hline ASA physical status (I/II/III) & $52 / 197 / 54$ & $51 / 144 / 51$ & 0.295 \\
\hline Duration of surgery (min) & $233.2 \pm 98.4$ & $225.9 \pm 84.4$ & 0.747 \\
\hline History of low back pain & 119 (39.3) & 97 (39.4) & 0.995 \\
\hline Position during the operation (supine/lithotomy) & $198 / 105$ & $159 / 87$ & 0.862 \\
\hline Type of operation (open/laparoscopy) & $173 / 130$ & $138 / 108$ & 0.814 \\
\hline Interventions & & & 0.799 \\
\hline Colon surgery & $156(51.5)$ & $127(51.6)$ & \\
\hline Stomach surgery & $107(35.3)$ & $87(35.4)$ & \\
\hline Liver surgery & $19(6.3)$ & $10(4.1)$ & \\
\hline Pancreaticoduodenectomy & $16(5.3)$ & $17(6.9)$ & \\
\hline Combined operation & $5(1.7)$ & $5(2.0)$ & \\
\hline
\end{tabular}

Values are expressed as number (\%), mean \pm SD, or number. Combined operation includes two or more abdominal organ surgery. ASA: American Society of Anesthesiologists.

Table 2. Measurements during Epidural Catheterization

\begin{tabular}{lccc}
\hline \multicolumn{1}{c}{ Variable } & Awake group & Anesthetized group \\
& $(\mathrm{n}=303)$ & $(\mathrm{n}=246)$ & P value \\
\hline The time for catheterization (min) & $1.6 \pm 1.5$ & $1.1 \pm 1.2$ & $1(1,2)$ \\
Number of attempts & $1(1,3)$ & $177(72.0) / 69(28.0)$ & 0.001 \\
The easiness of catheter insertion (easy/difficult) & $208(68.6) / 95(31.4)$ & $\mathrm{NA}$ & 0.4003 \\
Radicular pain & $5(1.7)$ & $2(0.8)$ & $\mathrm{NA}$ \\
Dural puncture & $3(1.0)$ & $32(13.0)$ & 0.999 \\
Vascular injury & $32(10.6)$ & 0.374 \\
\hline
\end{tabular}

Values are expressed as mean $\pm \mathrm{SD}$, median (1Q, 3Q), or number (\%). NA: not applicable. 
Table 3. Duration of Epidural Patient-controlled Analgesia and Postoperative Pain Score

\begin{tabular}{cccr}
\hline Variable & $\begin{array}{c}\text { Awake } \\
\text { group } \\
(\mathrm{n}=303)\end{array}$ & $\begin{array}{c}\text { Anesthetized } \\
\text { group } \\
(\mathrm{n}=246)\end{array}$ & P value \\
\hline PCA infusion duration $(\mathrm{h})$ & $\begin{array}{c}76.6 \pm 25.7 \\
78.3 \pm 20.7\end{array}$ & 0.070 \\
All surgeries & 303 & 246 & \\
Postoperative day 1 & $3(2,4)$ & $4(3,5)$ & $<0.001$ \\
Postoperative day 3 & $2(2,3)$ & $3(2,4)$ & 0.002 \\
Colon surgery & 156 & 127 & \\
Postoperative day 1 & $3(2,4)$ & $5(3,5)$ & $<0.001$ \\
Postoperative day 3 & $2(2,3)$ & $3(3,5)$ & 0.005 \\
Stomach surgery & 107 & 87 & \\
Postoperative day 1 & $3(2,5)$ & $4(3,5)$ & 0.001 \\
Postoperative day 3 & $2(2,3)$ & $3(2,4)$ & 0.055 \\
Liver surgery & 19 & 10 & \\
Postoperative day 1 & $3(3,4.5)$ & $4(2,5)$ & 0.795 \\
Postoperative day 3 & $2(2,3)$ & $2.5(1,4)$ & 0.854 \\
Pancreaticoduodenectomy & 16 & 17 & \\
Postoperative day 1 & $3(3,3.8)$ & $5(3,5)$ & 0.038 \\
Postoperative day 3 & $3(2,3.8)$ & $2(2,3.5)$ & 0.681 \\
\hline
\end{tabular}

Values are expressed as mean $\pm \mathrm{SD}$, number, or median $(1 \mathrm{Q}, 3 \mathrm{Q})$.

group (3 [2, 4] vs. $4[3,5]$ on postoperative day $1, \mathrm{P}<0.001$, and $2[2,3]$ vs. $3[2,4]$ on postoperative day $3, \mathrm{P}=0.002])$. The postoperative pain scores were significantly lower in the awake group compared to the anesthetized group on days 1 and 3 after colon surgery $(3[2,4]$ vs. $4[3,5]$ on postoperative day $1, \mathrm{P}$ $<0.001$ and $2[2,3]$ vs. $3[3,5]$ on postoperative day $3, \mathrm{P}=0.005)$. In addition, pain scores were significantly lower in the awake group compared to the anesthetized group on day 1 after stomach surgery $(3[2,5]$ vs. $4[3,5], \mathrm{P}=0.001)$ and pancreaticoduodenectomy $(3[3,3.8]$ vs. $5[3,5], \mathrm{P}=0.038)$ (Table 3 ).

Adverse events did not show an intergroup difference (Table 4). Serious neurological complications, such as meningitis, epidural abscess, epidural hematoma, spinal cord injury, spinal cord ischemia and paraplegia, were not observed in either group.

\section{DISCUSSION}

The optimal timing of epidural catheterization in adult patients is controversial. Epidural catheterization under general anesthesia can reduce patient discomfort and anxiety, but for several reasons it may also increase the incidence of serious complications. First, the reliability of an epidural test dose containing epinephrine, which is used to avoid the need for intravascular injection of local anesthetics, is reduced be-
Table 4. Adverse Events

\begin{tabular}{lccr}
\hline \multicolumn{1}{c}{ Event } & $\begin{array}{c}\text { Awake } \\
\text { group } \\
(\mathrm{n}=303)\end{array}$ & $\begin{array}{c}\text { Anesthetized } \\
\text { group } \\
(\mathrm{n}=246)\end{array}$ & P value \\
\hline Thoracic back pain & $22(7.3)$ & $20(8.1)$ & 0.703 \\
Maximal back pain (NRS) & $2(1,3)$ & $1(1,2.8)$ & 0.128 \\
Headache & $10(3.3)$ & $5(2.0)$ & 0.365 \\
Nausea & $8(2.6)$ & $6(2.4)$ & 0.882 \\
Vomiting & $2(0.7)$ & $1(0.4)$ & $>0.999$ \\
Urinary retention & $4(1.3)$ & $3(1.2)$ & $>0.999$ \\
Dizziness & $1(0.3)$ & $2(0.8)$ & 0.590 \\
Pruritus & $2(0.7)$ & $1(0.4)$ & $>0.999$ \\
Constipation & $0(0)$ & $1(0.4)$ & 0.448 \\
Paresthesia & $7(2.3)$ & $6(2.4)$ & 0.921 \\
Upper extremity & $1(0.3)$ & $2(0.8)$ & 0.590 \\
Lower extremity & $6(2.0)$ & $4(1.6)$ & $>0.999$ \\
Serious complications & $0(0)$ & $0(0)$ & NA \\
\hline
\end{tabular}

Values are expressed as number (\%) or median (1Q, 3Q). NRS: numerical rating scale $(0=$ no pain, 10 = worst pain imaginable), NA: not applicable. Serious complications include meningitis, epidural abscess, epidural hematoma, spinal cord injury, spinal cord ischemia and paraplegia in specified period.

cause the hemodynamic response varies by depth and type of anesthesia [10,11]. Furthermore, general anesthesia makes it difficult to differentiate between epidural and subarachnoid injection of local anesthetic, because the patient cannot complain of symptoms resulting from a sudden increase in the spinal anesthesia level, such as changes in consciousness. Second, the practitioner cannot obtain information about impending nerve injury from patients under general anesthesia; therefore, epidural placement while awake ameliorates concerns regarding several complications, especially neurological ones [8]. However, if the patient is uncooperative and requires epidural anesthesia, the practitioner can attempt the procedure with the patient in an anesthetized state.

There was a statistically significant difference in the total catheterization time between the awake and anesthetized patients in this study, with awake patients requiring more time for thoracic epidural catheterization. This result is considered to be due to the increased number of catheterization attempts in awake patients. The time required for epidural catheterization in awake patients is affected by various factors, such as patient anxiety, cooperation, position and pain. The most important factors in terms of procedure time in awake patients are patient mobility and cooperation (i.e., maintaining the proper position), where discomfort may arise from pain and anxiety during the procedure. Epidural 
placement under general anesthesia may provide a more comfortable environment for the practitioner, such that the number of placement attempts required may be reduced. Multiple catheterization attempts are closely associated with an increased risk of complications [12]. However, according to our results, even though the number of attempts increased in awake patients, this did not increase the incidence rates of complications such as dural puncture, vascular injuries or neurological complications. The incidence of dural puncture in this study was lower than that of a previous study (3.4\%), in which thoracic epidural catheterization was performed in the lower thoracic region of awake patients [13]. We expected a decreased incidence of dural puncture and easier placement of the catheter in the anesthetized state, due to the use of neuromuscular blocking agents that allowed a fixed and stable spinal position; however, no significant difference in dural puncture incidence was seen between the two groups.

Although the pain scores on postoperative day 1 were lower in the awake group than in the anesthetized group after colon surgery, stomach surgery, and pancreaticoduodenectomy, this result requires further investigation. Pain level differs by type of surgery (e.g., open vs. laparoscopic) and extent of operation (e.g., presence vs. absence of radical lymph node dissection). In addition, postoperative pain was internally controlled by the surgeon and various analgesics were injected irrespective of patient-controlled epidural analgesia; these factors could have affected the accuracy of the postoperative pain score analyses. Furthermore, correct placement of the epidural catheter was not confirmed by fluoroscopy, but only by indirect anatomical assessments (LOR, surface measurements of depth, palpation). When epidural catheterization is performed with a paramedian approach, the catheter is more likely to enter through the lateral side rather than the midline [14], which may also affect the degree of analgesia provided by epidural PCA. However, combining thoracic epidural analgesia and general anesthesia is advantageous for postoperative care, conferring more effective analgesia and reducing stress hormone levels relative to general anesthesia alone [3]. Although thoracic epidural analgesia carries a lower risk of complications than other perioperative treatments [2], complications related to thoracic epidural catheterization can be catastrophic. Complaints including radicular pain may arise from mechanical stimulation of the spinal nerve root or cord by the epidural needle or catheter, serving as an early warning sign before serious neurological complications occur $[15,16]$. Thus, such complaints should not be ignored, and the epidural needle or catheter should be withdrawn if they are present. Additional treatment is not usually necessary and neurological sequelae seldom occur after catheter withdrawal [13]. Thoracic epidural catheterization is not generally recommended under general anesthesia in patients who have previously undergone spinal surgery at the epidural catheterization site, because identification of the epidural space would be difficult due to the obliteration of predictable surface and anatomical landmarks [15]. Serious nerve or cord injury may occur in patients under anesthesia or deep sedation because of an absence of feedback regarding radicular pain. Therefore, thoracic epidural catheterization under general anesthesia should be performed carefully, and only when necessary.

The possibility of traumatic puncture may increase due to degenerative changes of the spine and reduced epidural space in elderly patients [2]. Moreover, early detection and proper management of neurological injury could be impeded by increased vulnerability to residual anesthetic effects and mental deterioration in elderly patients [17]. The majority of serious complications occur in elderly patients with comorbidities, regardless of the neuraxial technique applied [18]. Therefore, in these patients, performing epidural catheterization while awake, or offering alternative postoperative pain control methods such as intravenous PCA, seems to be appropriate for avoiding serious complications.

Lumbar epidural catheterization can be performed with a midline approach without fluoroscopy guidance, because the anatomy of the lumbar spine is relatively clear. Epidural catheterization is expected to be more technically difficult in the thoracic region, which could increase the incidence of epidural catheterization-related neurological complications in the thoracic region compared to the lumbar region. However, in 4,185 subjects, Giebler et al. [13] showed that the incidence and predicted risk of permanent neurological complications after thoracic epidural catheterization were $3.1 \%$ and $0.07 \%$, respectively. The authors concluded that complications after thoracic epidural catheterization may not be increased after lumbar epidural catheterization [13]. In accordance with this, only mild neurological complications, such as headache, dizziness, and paresthesia, were observed in the present study; there were no permanent or serious complications caused by 
thoracic epidural catheterization.

The overall incidence and number of maximum NRS back pain scores were not significantly different between the two groups in this study. Our expectation was that the incidence of, and scores for, back pain would decrease when thoracic epidural catheterization was conducted after anesthesia because of patient immobility, reduced time required for the procedure, and reduced rate of procedure-related back tissue injury. However, lidocaine injection, which can serve as preemptive analgesia [19], was administered at the puncture site only in the awake patient group and likely influenced the level of back pain. A previous study recorded a $26.7 \%$ incidence of back pain after lumbar epidural catheterization with a midline approach in non-obstetric surgery patients [20]. In contrast, the incidence of back pain was only $7.3 \%$ in awake patients and $8.1 \%$ in anesthetized patients in our study. Although direct comparison is difficult because of the different regions targeted, the decreased back pain incidence in our study might have resulted from the use of a paramedian approach for thoracic epidural catheterization. It is possible that the midline approach can cause more damage to the interspinous ligaments, and thus result in greater levels of subsequent back pain, than the paramedian approach.

This study had some limitations. First, although more than 500 thoracic epidural catheterization procedures were analyzed according to the presence or absence of anesthesia at the time of catheterization, the sample size was too small to effectively compare the incidence of complications; thus, it is possible that no significant difference in the rate of complications existed between the two groups. Second, the retrospective nature of the study precluded long-term followup; only complications that occurred during the hospital stay were documented and analyzed. If extended follow-up observations were performed, the incidence of complications in both groups may have been different. Third, all patients in this study underwent epidural catheterization in the lowerthoracic region (T9-12 level). The rate of complications would likely have been different if upper- and mid-thoracic epidural catheterizations were included, as dural perforation and catheter-related complications were more common in the lower- than mid- or upper-thoracic region in a previous study [13]. In this study, vascular injury during epidural catheterization was determined by the absence of blood in a Tuohy needle hub or negative blood aspiration from the
Tuohy needle or epidural catheter. However, this approach is unreliable for detecting intravascular needle placement [21] and may underestimate the incidence of vascular injury compared to evaluations based on digital subtraction fluoroscopic imaging $[22,23]$. Finally, use of the lateral decubitus position for epidural catheterization can result in an increase in peak inspiratory pressure and hemodynamic changes, such as increased heart rate decreased mean arterial pressure, in anesthetized patients [24,25]. However, such physiological changes, which may be caused by lateral decubitus positioning during epidural catheterization, were not assessed in this study.

In conclusion, thoracic epidural catheterization in awake patients requires more attempts for successful catheterization versus anesthetized patients, but the risk of complications does not differ by timing of implementation of the procedure. However, long-term follow-up studies in larger populations are needed to ensure the safety of this procedure in anesthetized adult patients. The hazards of thoracic epidural catheterization posed to anesthetized patients, in particular serious neurological injury, , as well as the benefits, must be considered by clinicians despite the extremely rare occurrence of serious neurological complications.

\section{CONFLICTS OF INTEREST}

No potential conflict of interest relevant to this article was reported.

\section{ORCID}

Seok-Jin Lee: https://orcid.org/0000-0001-7894-8510

Sung-Ae Cho: https://orcid.org/0000-0002-1519-3787

Chi Bum In: https://orcid.org/0000-0003-3658-5277

Po-Soon Kang: https://orcid.org/0000-0002-5000-1517

\section{REFERENCES}

1. Bromage PR, Camporesi E, Chestnut D. Epidural narcotics for postoperative analgesia. Anesth Analg 1980; 59: 473-80.

2. Freise H, Van Aken HK. Risks and benefits of thoracic epidural anaesthesia. Br J Anaesth 2011; 107: 859-68.

3. Guay J. The benefits of adding epidural analgesia to general anesthesia: a metaanalysis. J Anesth 2006; 20: 335-40.

4. Radovanović D, Radovanović Z, Škorić-Jokić S, Tatić M, Mandić A, 
Ivković-Kapicl T. Thoracic epidural versus intravenous patientcontrolled analgesia after open colorectal cancer surgery. Acta Clin Croat 2017; 56: 244-54.

5. Wu CL, Rowlingson AJ, Herbert R, Richman JM, Andrews RA, Fleisher LA. Correlation of postoperative epidural analgesia on morbidity and mortality after colectomy in Medicare patients. J Clin Anesth 2006; 18: 594-9.

6. Giaufre E, Dalens B, Gombert A. Epidemiology and morbidity of regional anesthesia in children: a one-year prospective survey of the French-Language Society of Pediatric Anesthesiologists. Anesth Analg 1996; 83: 904-12.

7. Taenzer AH, Walker BJ, Bosenberg AT, Martin L, Suresh S, Polaner DM, et al. Asleep versus awake: does it matter?: pediatric regional block complications by patient state: a report from the Pediatric Regional Anesthesia Network. Reg Anesth Pain Med 2014; 39: 279-83.

8. Rosenquist RW, Birnbach DJ. Epidural insertion in anesthetized adults: will your patients thank you? Anesth Analg 2003; 96: 1545-6.

9. Horlocker TT, Abel MD, Messick JM Jr, Schroeder DR. Small risk of serious neurologic complications related to lumbar epidural catheter placement in anesthetized patients. Anesth Analg 2003; 96: 1547-52.

10. Liu SS, Carpenter RL. Hemodynamic responses to intravascular injection of epinephrine-containing epidural test doses in adults during general anesthesia. Anesthesiology 1996; 84: 81-7.

11. Guay J. The epidural test dose: a review. Anesth Analg 2006; 102: 921-9.

12. Cheney FW, Domino KB, Caplan RA, Posner K. Nerve injury associated with anesthesia: a closed claims analysis. Anesthesiology 1999; 90: 1062-9.

13. Giebler RM, Scherer RU, Peters J. Incidence of neurologic complications related to thoracic epidural catheterization. Anesthesiology 1997; 86: 55-63.

14. Sharrock NE, Go G. Lateralizing epidural catheter placement as evidenced by electrical stimulation. Clin Orthop Relat Res 2014; 472: 1449-52.
15. Krane EJ, Dalens BJ, Murat I, Murrell D. The safety of epidurals placed during general anesthesia. Reg Anesth Pain Med 1998; 23 : 433-8.

16. Bromage PR, Benumof JL. Paraplegia following intracord injection during attempted epidural anesthesia under general anesthesia. Reg Anesth Pain Med 1998; 23: 104-7.

17. Kao MC, Tsai SK, Tsou MY, Lee HK, Guo WH, Hu JS. Paraplegia after delayed detection of inadvertent spinal cord injury during thoracic epidural catheterization in an anesthetized elderly patient. Anesth Analg 2004; 99: 580-3.

18. Pitkänen MT, Aromaa U, Cozanitis DA, Förster JG. Serious complications associated with spinal and epidural anaesthesia in Finland from 2000 to 2009. Acta Anaesthesiol Scand 2013; 57: 553-64.

19. Lowenstein L, Zimmer EZ, Deutsch M, Paz Y, Yaniv D, Jakovi P. Preoperative analgesia with local lidocaine infiltration for abdominal hysterectomy pain management. Eur J Obstet Gynecol Reprod Biol 2008; 136: 239-42.

20. Chan ST. Incidence of back pain after lumbar epidural anesthesia for non-obstetric surgery--a preliminary report. Med J Malaysia 1995; 50: 241-5.

21. Furman MB, Giovanniello MT, O'Brien EM. Incidence of intravascular penetration in transforaminal cervical epidural steroid injections. Spine (Phila Pa 1976) 2003; 28: 21-5.

22. Lee MH, Yang KS, Kim YH, Jung HD, Lim SJ, Moon DE. Accuracy of live fluoroscopy to detect intravascular injection during lumbar transforaminal epidural injections. Korean J Pain 2010; 23: 18-23.

23. Park Sk, Choi YS. Advantages of digital subtraction angiography during nerve block. Anesth Pain Med 2014; 4: e19927.

24. Tanskanen P, Kyttä J, Randell T. The effect of patient positioning on dynamic lung compliance. Acta Anaesthesiol Scand 1997; 41: 602-6.

25. Eggers GWN Jr, deGroot WJ, Tanner CR, Leonard JJ. Hemodynamic changes associated with various surgical positions. JAMA 1963; 185: 1-5. 